\title{
Validation of electronic diary (PRO-Diary) compared to validated paper questionnaires in normal individuals
}

\author{
S. Hampton and B. Middleton \\ Faculty of Health and Medical Sciences, University of Surrey, Guildford, Surrey GU2 7XH, UK
}

The use of electronic devices such as hand-held personal digital assistants (PDA) to record food frequency questionnaires, $24 \mathrm{~h}$ recalls and dietary history is in the early stages of development and has been recently reviewed ${ }^{(1)}$. However, the literature on using such digital devices to measure food visual analogue scales (VAS) is scant. This study investigated the use of electronic recording in the form of PRO-Diary $v$. paper food VAS responses after consuming a cereal bar in the morning. The aim of the study was to establish that food VAS responses recorded on the PRO-Diary could be used instead of the paper questionnaires.

Fifty healthy participants $(19 \mathrm{~F}, 31 \mathrm{M})$ mean age 31 years $(11.5 \mathrm{SD})$ with a BMI $22 \mathrm{~kg} / \mathrm{m}^{2}$ took part in a controlled randomised, single blind crossover study. Participants were randomised into groups A and B, group A participants were asked to record food VAS scores using the PRODiary on the first occasion and on the second visit on paper. Group B participants were asked to complete the protocol in reverse. The PRO-Diary questions were set to occur randomly and therefore the paper questionnaires were also randomised. There were at least $2 \mathrm{~d}$ between each study visit. Participants were given a standardised meal $460 \mathrm{kcal}(1924.64 \mathrm{~kJ})$ the evening prior to attending the suite. Nothing was eaten or drunk except water until they arrived in the suite. On arrival in the suite, participants were asked to complete a food VAS questionnaire at time 0. Participants were given a cereal bar $88 \mathrm{kcal}(368.2 \mathrm{~kJ})$ plus $200 \mathrm{ml}$ water at 09.00 hours. They completed food VAS scales at $15,30,45,60,75$ and 90 min after consuming the bar. The food VAS paper questionnaires were manually scored and entered into a spreadsheet. The PRO-Dairy data was downloaded and transferred to an Excel spreadsheet. Statistical analysis was undertaken on the raw data and the calculated differences between the digital and paper results. All VAS data generated from the study was analysed using two-way repeated measures ANOVA (factors, time and treatment). Any differences were identified with Tukey's post hoc tests with $P<0.05$ regarded as significant.

Two way RM-ANOVA showed a significant effect of time for all types of food VAS questionnaires. No significant differences were observed between the PRO-Dairy and the paper food VAS questionnaire except for how full the participant felt (Figure). Participants were significantly less hungry when they used the PRO-Dairy than when they used paper $(P=0.04)$. The difference between PRO-Dairy and manual data was calculated to establish correlation by plotting the difference and including $95 \%$ confidence limits at each time point for - how hungry do you feel question. Only the last two time points did the $95 \%$ confidence limit fail to cross the $X$-axis.

Overall there were no differences in the food VAS scoring for all criteria except how full do you feel and only after 60 min. However, the PRODairy data returned to baseline levels, whereas the paper diary showed responses well below baseline levels. So it could be argued that the PRODairy gave accurate results.

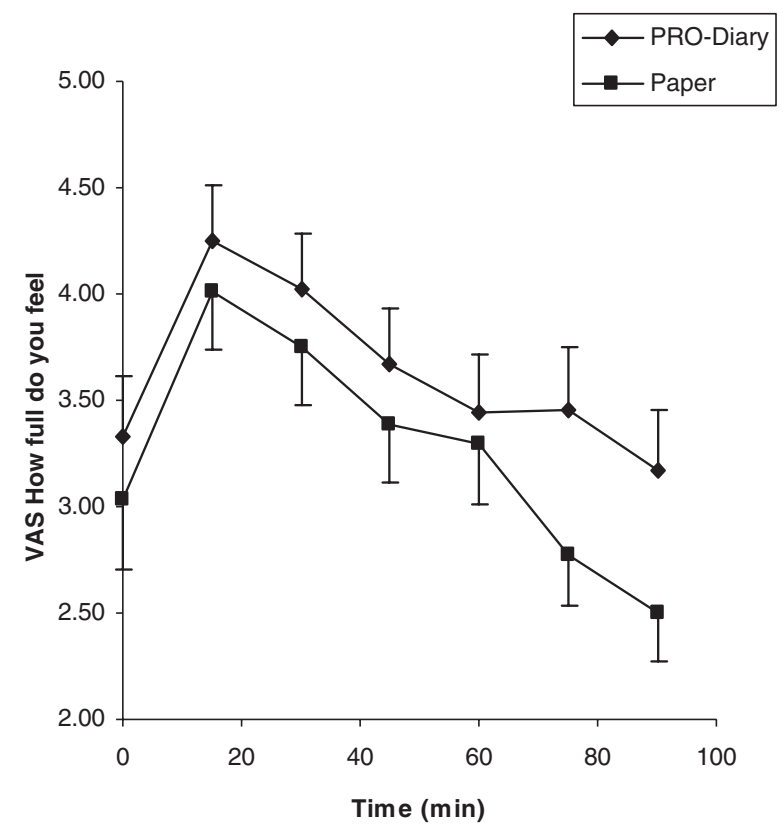

1. Ngo J, Engelen A, Molag M et al. (2009) Br J Nutr 101, Suppl 2, S102-S112. 